\title{
Cross-National Analysis on Sensitivity to Environmental Quality and Its Change in East Asia
}

\author{
Yanyan Chen', Yuejun Zheng² \\ ${ }^{1}$ Graduate School of Culture and Information Science, Doshisha University, Kyoto, Japan \\ ${ }^{2}$ Faculty of Culture and Information Science, Doshisha University, Kyoto, Japan \\ Email: ein1003@mail4.doshisha.ac.jp, yzheng@mail.doshisha.ac.jp
}

Received 23 May 2015; accepted 16 June 2015; published 19 June 2015

Copyright (C) 2015 by authors and Scientific Research Publishing Inc.

This work is licensed under the Creative Commons Attribution International License (CC BY). http://creativecommons.org/licenses/by/4.0/

c) (7) Open Access

\begin{abstract}
This paper aims to compare people's sensitivity to environmental change in China, Japan and South Korea across spatial and temporal dimensions by analysing the data derived from a crossnational survey. Based on the analytical results derived from 3907 samples, the consistency of people's environmental sensitivity on the temporal dimension and the gap on the spatial dimension are clarified. The analysis on the temporal dimension indicates that people are inclined to hold a consistent evaluation toward environmental change, while the analysis on the spatial dimension of environmental sensitivity indicates that people are more sensitive to the deterioration of global rather than domestic environmental change. Data analysis also indicates that Japanese and South Koreans are pessimistic regarding the change of the environment while the Chinese are optimistic. In addition, the attributive features of individuals who are more sensitive to the environmental change are clarified within each country, and the results show that under different social backgrounds, the influence of demographic factors is somewhat dissimilar.
\end{abstract}

\section{Keywords}

Environmental Consciousness, Environmental Evaluation, Spatial-Temporal Dimension, Categorical Data Analysis

\section{Introduction}

Since the beginning of the 1960s, environmental issues have become a global topic and have received more and more attention. The practice of environmental conservation has proven that environmental issues are not only a 
technological or economic issue, but also a social issue. It is, as the final consequence, a result of a "crisis" in the values of people (Disch, 1970; Swan, 1971). Therefore, the solution to environmental degradation requires the adjustment of values and the improvement of environmental consciousness.

However, environmental consciousness is complicated and comprehensive. The study of environmental consciousness has a history of nearly 50 years since the concept first emerged in the late 1960s (Roth, 1968). However, how to define environmental consciousness remains unclear. Some studies have taken a "paradigm or value shift" perspective and have proposed that environmental consciousness represents a new world view and reflects a new way of thinking (Dunlap \& Van Liere, 1978; Inglehart, 1997); some studies have defined environmental consciousness as a function of value orientations, such as egoism, altruism or other deeper causes (Stern, 1992; Axelrod, 1994; Merchant, 1992). There are also some studies that have attempted to clarify and refine this ambiguous definition from a general perspective which includes nominal, functional and operational environmental literacy (Roth, 1991).

According to Zheng (2010), environmental consciousness is defined as a kind of mental behaviour that reflects the individual's recognition, value judgment and behavior intention toward environmental issues. In most situations, it implies the individual's subjective cognition, perception and value judgment on the history, present state, and change in environmental state identified by a specific spatial and temporal context. In this definition, people's subjective response to the environmental change as well as the spatial-temporal context is emphasized. Environmental consciousness, in essence, is people's subjective feeling and judgment toward the specific surrounding environment. People's subjective responses to the objective environment are the inherent content of environmental consciousness. With the changing of the spatial-temporal dimension in which individuals are embedded, people's responses are often different. Based on this definition, this paper tries to compare people's subjective responses to environmental change from a perspective of environmental sensitivity.

Environmental sensitivity in this paper is defined as people's sensibility to perceiving changes to environmental quality, as well as the evaluation on such change. Sensibility concerns with the issue whether individuals perceive the change of environmental quality. Evaluation relates to the issue of how individuals evaluate such change, that is, whether they believe the environmental quality gets better (positive evaluation) or turns worse (negative evaluation). Environmental sensitivity is a self-centred formation which is based on personal experience and determined by individuals' cognition and personality. Hence, even to the same environmental quality or change, diversified responses may be given. The analysis on environmental sensitivity will benefit our understanding of the formation of environmental consciousness on a personal level, as well as ways to improve people's environmental consciousness. Furthermore, individuals are living in a society where different attitudes toward environmental issues interact. People's environmental evaluation is inevitably affected by other people and hence represents a social facet (Zheng, 2012). The analysis on environmental sensitivity of different societies will benefit our knowledge regarding the influence of social structures to people's environmental consciousness, as well as the mutual understanding and cooperation on environmental conservation of these areas. China, Japan, and South Korea are the main countries in East Asia with different social backgrounds and characteristics. In the past few decades, environmental quality in these three countries has changed to some degree. People's sensitivity to these changes within one country, and the national response features to these changes are our concerns.

In order to analyse people's environmental sensitivity in these three countries, spatial and temporal dimensions are referred to in this study. The spatial dimension is used to investigate people's environmental sensitivity on local and global environmental quality and change, while the temporal dimension is used to investigate people's environmental sensitivity in a given time frame, which includes the perception of environmental change in the past, the satisfaction with the environmental quality in the present, and the prediction of environmental issues in the future. The main purpose of this study is to explore the common feature as well as the traits of environmental sensitivity in China, Japan and South Korea from the spatial and temporal dimensions by analysing the data from a cross-national survey. Furthermore, the attributive features of individuals who are more sensitive to the environmental change are also clarified within one country.

\section{Measurement and Method}

The data used in this paper was collected from a cross-national survey entitled "The East Asian Survey on People's Sense of Culture, Life and Environment (2011)” which was conducted by Doshisha Research Center for East Asian Studies. A carefully designed questionnaire was used by the investigators to interview respon- 
dents who were scientifically selected by the multistage sampling in China, Japan and South Korea (more detailed information see Table 1). Environmental consciousness in adults is supposed to be more stable than with the minors. According to the age of majority recognized by the laws in the three countries, this survey took Japanese citizens who are 20 years or older, South Korean citizens who are 19 years or older and Chinese citizens who are 18 years or older as the target population. The survey was conducted in 47 prefectures of Japan and 894 valid samples were obtained. The survey in South Korea was conducted nationwide, with the exception of Jeju Island. 1002 adult South Korean citizens were interviewed successfully. Considering the vast, heterogeneous population of China, two cities, Beijing and Hangzhou, were selected as survey areas to represent northern and southern China, respectively. Chinese adult citizens living in these two cities were selected and 1000 valid samples in Beijing and 1011 valid samples in Hangzhou were collected respectively.

In the survey, we used Q12 to examine people's sensitivity to global environmental change, and Q13 to examine people's sensitivity to domestic environmental change in the past several years. Q7 is used to investigate people's satisfaction with current environmental quality, including the clearness of air and water, the lushness of fauna (i.e., vegetation, forests), as well as the comfort level of the living conditions. Q9 is used to investigate people's prediction regarding several domestic environmental issues in the future. The above question items are elaborated in detail in Table 2. Furthermore, demographic factors such as gender, age, educational level, and yearly household income are also introduced, in order to verify the relationship between social attributes and environmental sensitivity.

The multiple correspondence analysis (MCA) method is statistical technique for high-dimensional categorical data which allows us to analyse the pattern of relationships among more than three categorical variables (Zheng, 2010). In order to clarify the relationship among different categorical variables, the MCA method is used in this paper.

\section{Results}

In this section, we first compare people's sensitivity to global and domestic environmental changes based on the spatial dimension. We then analyse the people's environmental sensitivity by a time series, which includes perception of environmental change in the past, satisfaction with the environmental quality in the present, and prediction of environmental issues in the future. Finally, the relationship between demographic factors and people's perception on environmental change is visually analysed in three countries. In order to clarify the potential features of environmental consciousness in northern and southern China, we focus on the results of Beijing andHangzhou respectively, instead of China as whole.

\subsection{Environmental Sensitivity on the Spatial Dimension}

In the survey, respondents were asked whether they feel the global environment and domestic environment improved or got worse in the past several years. The responses are shown in Figure 1 and Figure 2.

From Figure 1, we found that respondents in Japan and South Korea are inclined to give negative evaluations on the change of global environmental quality in the past, while respondents in both of Chinese cities are inclined to hold positive evaluations. 68.2\% respondents in Japan and 70.6\% in South Korea believed the global environment in general got worse, while only a small part (8.1\% in Japan and $11.5 \%$ in South Korea) of the

Table 1. Sample design, sample mode and completed interviews.

\begin{tabular}{ccccc}
\hline & Japan & South Korea & Beijing & Hangzhou \\
\hline Study population & Over 20 years & Over 19 years & Over 18 years & Over 18 years \\
Sample & 2-stage sampling & 3-stage sampling & 2-stage sampling & 2-stage sampling \\
Survey mode & & Face to face interview & 1000 (quota) \\
Target number of samples & 1800 & 1000 (quota) & 1000 (quota) & 1011 \\
Completed interviews & 894 & 1002 & 1000 & $2011 / 10 / 13-25$ \\
\hline
\end{tabular}


Table 2. Related question items in the survey.

\begin{tabular}{|c|c|c|c|c|}
\hline Dimension & Number & Item Name & Question & Answer \\
\hline $\begin{array}{c}\text { Spatial } \\
\text { dimension }\end{array}$ & Q12 & $\begin{array}{l}\text { Global } \\
\text { environmental } \\
\text { change }\end{array}$ & $\begin{array}{l}\text { When you look at conditions around you as a whole in terms } \\
\text { of things like quality of the air, water, soil and the plants and } \\
\text { animals, do you think that environment on earth in general } \\
\text { has improved over the last several years, or do you think that } \\
\text { it has gotten worse? }\end{array}$ & $\begin{array}{l}\text { 1) Improved } \\
\text { 2) Improved somewhat } \\
\text { 3) No change } \\
\text { 4) Worsened somewhat } \\
\text { 5) Worsened }\end{array}$ \\
\hline \multirow{3}{*}{$\begin{array}{c}\text { Temporal } \\
\text { dimension }\end{array}$} & Q13 & $\begin{array}{l}\text { Domestic } \\
\text { environmental } \\
\text { change } \\
\text { (past perception) }\end{array}$ & $\begin{array}{l}\text { Then, do you think that the environment in your country } \\
\text { as a whole has improved over the last several years, or do } \\
\text { you think that it has gotten worse? }\end{array}$ & $\begin{array}{l}\text { 1) Improved } \\
\text { 2) Improved somewhat } \\
\text { 3) No change } \\
\text { 4) Worsened somewhat } \\
\text { 5) Worsened }\end{array}$ \\
\hline & Q7 & Current satisfaction & $\begin{array}{l}\text { How satisfied are you with quality of the following } \\
\text { environmental elements nearby your home? } \\
\text { 1) Cleanness of the air. } \\
\text { 2) Cleanness of the water (i.e., rivers or sea near your home). } \\
\text { 3) Lushness of fauna (i.e., vegetation, forests). } \\
\text { 4) Comfort level of your residence. }\end{array}$ & $\begin{array}{l}\text { 1) Satisfied } \\
\text { 2) Satisfied somewhat } \\
\text { 3) Dissatisfied somewhat } \\
\text { 4) Dissatisfied }\end{array}$ \\
\hline & Q9 & Future prediction & $\begin{array}{l}\text { In your country, do you think the following kinds of } \\
\text { environmental issues will get better in the next five years } \\
\text { or do you think they will get worse? } \\
\text { 1) Air pollution. } \\
\text { 2) Water contamination. } \\
\text { 3) Decline in forestry and vegetation. } \\
\text { 4) Degradation of food safety. } \\
\text { 5) Increase in the volume of garbage from homes. } \\
\text { 6) Increase in the volume of industrial waste. }\end{array}$ & $\begin{array}{l}\text { 1) Improve dramatically } \\
\text { 2) Improve } \\
\text { 3) No change } \\
\text { 4) Get worse } \\
\text { 5) Get worse dramatically }\end{array}$ \\
\hline
\end{tabular}

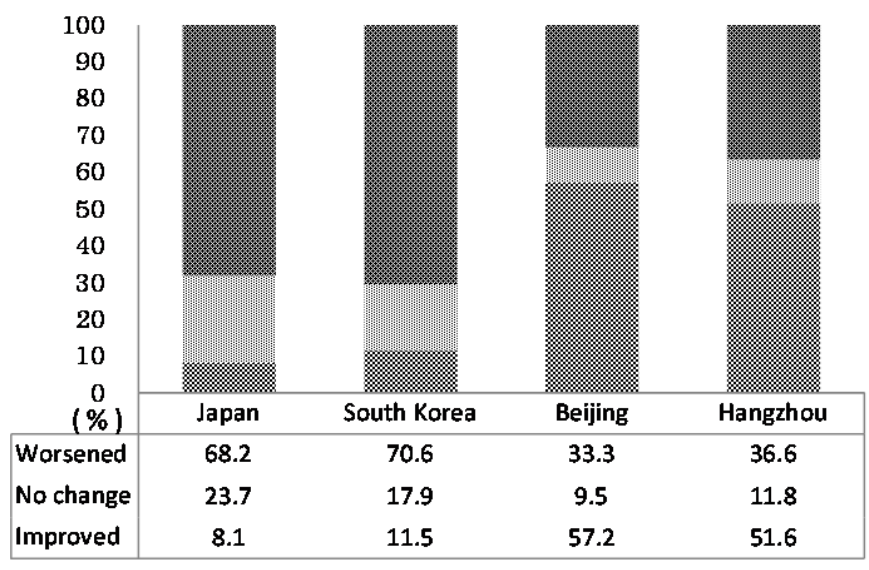

Figure 1. Sensitivity to global environmental change.

respondents in these two countries believed it had improved. To contrast, 57.2\% respondents in Beijing and 51.6\% in Hangzhou believed that global environmental quality had improved. Similarly, the negative evaluation in Japan and South Korea and the positive evaluation in Beijing and Hangzhou are also shown in the evaluation on domestic environmental change (see Figure 2).

However, although similarities were shown in the evaluations on both global and domestic environmental change, the degree of negative or positive evaluation on global and domestic environment proved to be different. In actuality, respondents in all three countries were inclined to give a more positive evaluation on the domestic environmental change than the global one (see Figure 3). Most Japanese and South Koreans believe that both the global and domestic environments got worse, yet they hold more negative evaluation regarding global change. Most respondents in Beijing and Hangzhou believe both the global and domestic environment had improved, yet they hold a more positive evaluation regarding domestic change. Hereby, we generally clarify the 


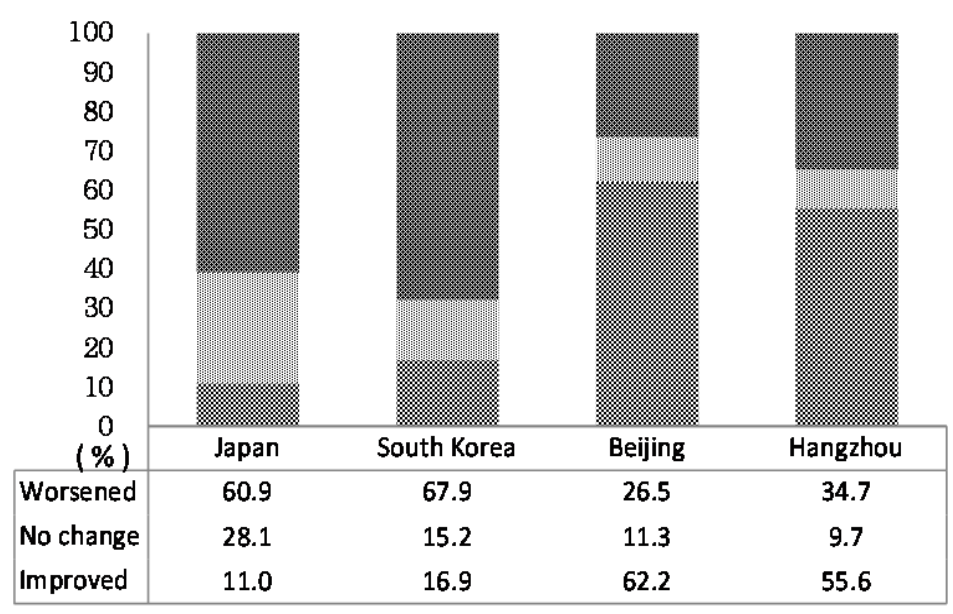

Figure 2. Sensitivity to domestic environmental change.

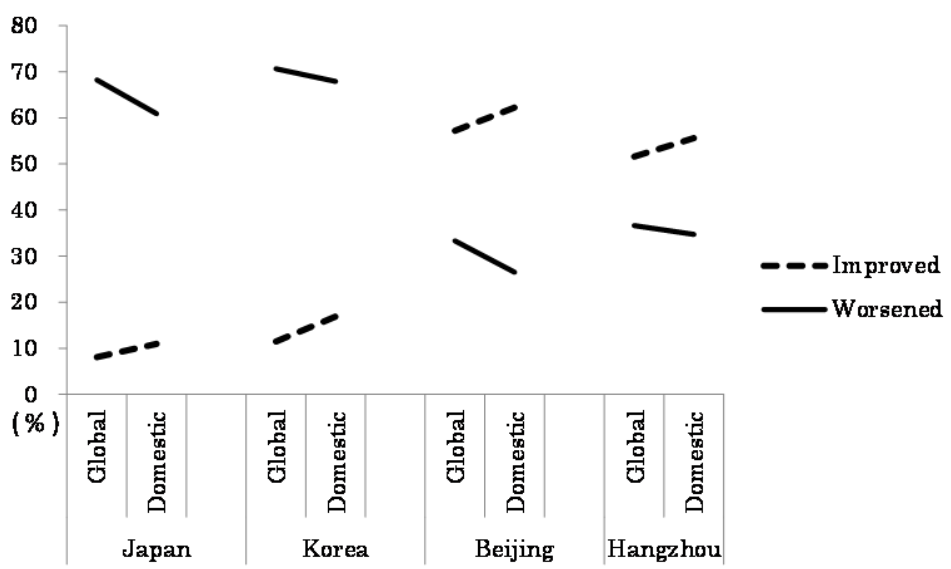

Figure 3. Relationship between evaluations on global and domestic environmental change.

spatial feature of environmental sensitivity in three countries that people are inclined to give a more positive evaluation to the domestic environmental change than the global change, which also indicates that people are more sensitive to the deterioration of the global environment than the domestic environment.

\subsection{Environmental Sensitivity on the Temporal Dimension}

The evaluation on environmental change in the past was clarified above. This is also taken as a starting point to conduct the analysis on the temporal dimension. In this section, satisfaction with the environmental quality in the present and the prediction of environmental issues in the future are analysed respectively. The relationship among environmental sensitivity on three temporal scales (past, current and future) is then clarified by the MCA method.

\subsubsection{Environmental Satisfaction}

In the survey, the respondents were asked to describe their satisfaction with the quality of local environmental elements including air purity, water quality, lushness of fauna and comfort level of living conditions. The responses from the three countries are shown in Table 3.

From the table, we found that more than half of the people in all three countries are satisfied or somewhat satisfied with all the environmental elements that we investigated in the survey. Although the Japanese show a negative evaluation on environmental change in the past, they express a very positive evaluation on environmental quality in the present. For all four environmental elements, the Japanese showed the highest percentages 
Table 3. Percentage of satisfaction with environmental quality.

\begin{tabular}{|c|c|c|c|c|c|}
\hline & & (\%) Japan & South Korea & Beijing & Hangzhou \\
\hline \multirow{4}{*}{ Air } & Satisfied & 40.2 & 19.4 & 13.7 & 24.5 \\
\hline & Somewhat satisfied & 48.2 & 43.8 & 46.5 & 54.5 \\
\hline & Somewhat dissatisfied & 10.1 & 26.8 & 27.7 & 17.9 \\
\hline & Dissatisfied & 1.6 & 10.0 & 12.1 & 3.2 \\
\hline \multirow{4}{*}{ Water } & Satisfied & 28.4 & 14.7 & 15.5 & 22.8 \\
\hline & Somewhat satisfied & 48.2 & 47.4 & 43.2 & 56.4 \\
\hline & Somewhat dissatisfied & 20.1 & 28.0 & 27.2 & 16.5 \\
\hline & Dissatisfied & 3.3 & 9.9 & 14.1 & 4.4 \\
\hline \multirow{4}{*}{ Nature } & Satisfied & 38.5 & 16.9 & 21.1 & 30.8 \\
\hline & Somewhat satisfied & 42.0 & 42.1 & 44.2 & 54.7 \\
\hline & Somewhat dissatisfied & 17.8 & 29.4 & 23.3 & 13.2 \\
\hline & Dissatisfied & 1.7 & 11.6 & 11.3 & 1.4 \\
\hline \multirow{4}{*}{$\begin{array}{l}\text { Living } \\
\text { Condition }\end{array}$} & Satisfied & 33.7 & 16.2 & 16.7 & 29.6 \\
\hline & Somewhat satisfied & 50.5 & 46.6 & 50.7 & 57.4 \\
\hline & Somewhat dissatisfied & 13.2 & 28.1 & 20.8 & 11.2 \\
\hline & Dissatisfied & 2.6 & 9.1 & 11.7 & 1.8 \\
\hline
\end{tabular}

of the "Satisfied" level ( $40.2 \%$ on air, $28.4 \%$ on water, $38.5 \%$ on nature and $33.7 \%$ on living environment). Together with the option of "Somewhat satisfied", more than $80 \%$ of the Japanese on average were satisfied or somewhat satisfied with the local environmental quality. Respondents in Hangzhou also showed a very high satisfaction with the environmental quality. Although their percentages of the "satisfied" option were somewhat lower than in Japan, more than $80 \%$ of respondents in Hangzhou showed "satisfied" or "somewhat satisfied" attitudes regarding environmental quality in the present. Compared to Japan and Hangzhou, respondents in South Korea and Beijing were more inclined to give a negative evaluation of and hold more dissatisfaction toward environmental quality of the present.

\subsubsection{Environmental Prediction}

In the survey, respondents were also asked to predict changes regarding domestic environmental issues, such as air pollution, water contamination, forestry declination, food safety, and the increase of household waste and industrial waste, in the next 5 years. The predictions of respondents in all three countries are shown in Figures 4(a)-(f).

From the figures, we found that the Japanese and South Koreans are more pessimistic, while the Chinese are optimistic regarding environmental change in the future. Regarding the environmental change in the future, the Japanese generally hold cautious expectations. Regarding the environmental issues we surveyed, nearly half of the Japanese respondents believed that these issues will get even worse, while only a very small part of people believed that these issues will improve. It should be noted that there is also considerable part of people who believe the issues with the environment will not change in the future. In South Korea, except for food safety issue, more than half of the respondents believed that the mentioned domestic environmental issues will get even worse in the future. The Chinese showed more positive expectations regarding change of environment issues in the future. Nearly half of the respondents in Beijing and Hangzhou believed that the environmental issues will improve in the future.

Regarding the environmental issues we surveyed, we found that in all three countries people has the least amount of confidence in changes to industrial waste. More than $60 \%$ of respondents in both Japan and South 


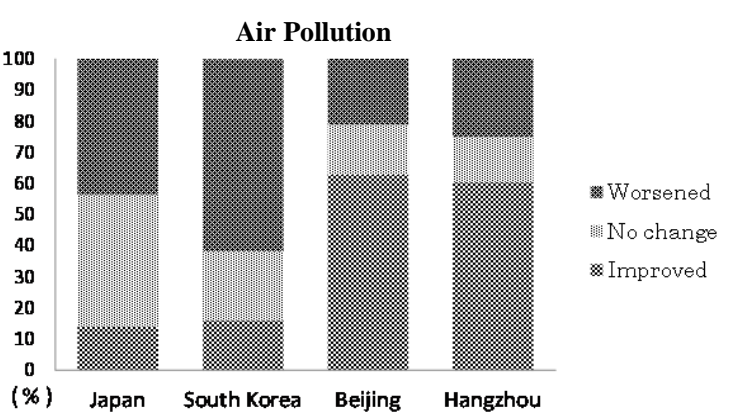

(a)

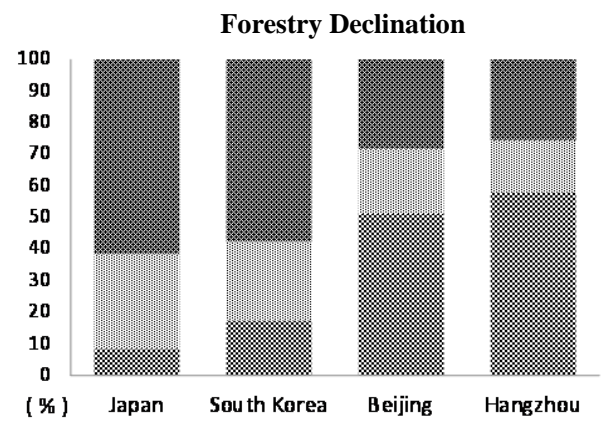

(c)

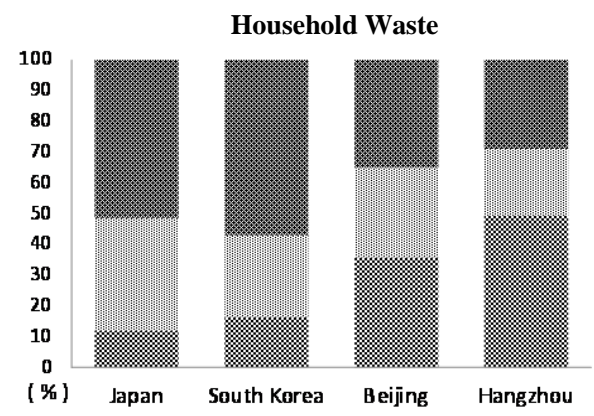

(e)

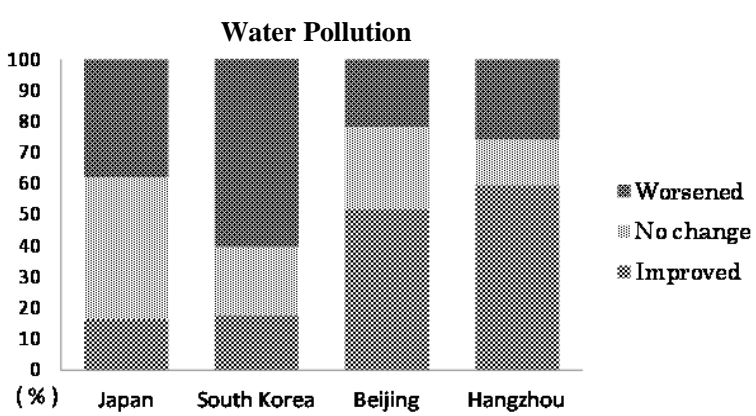

(b)

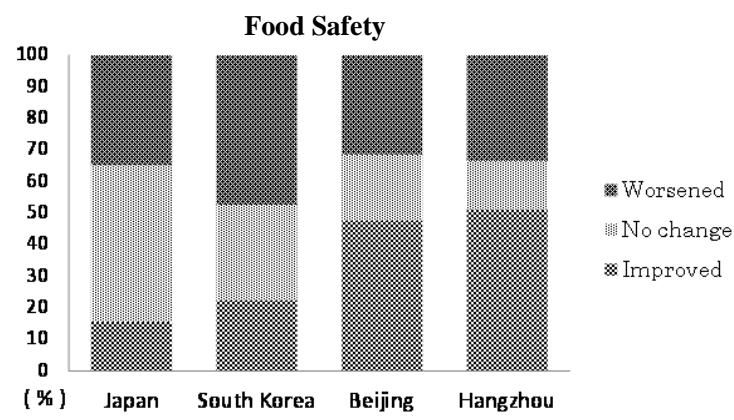

(d)

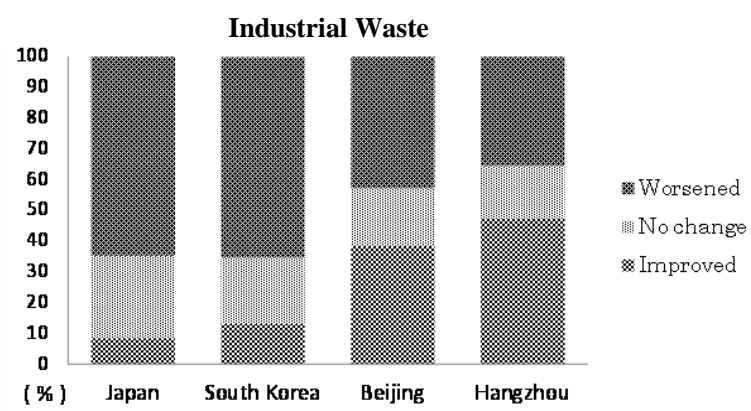

(f)

Figure 4. Prediction for domestic environmental issues of the future.

Korea believe that industrial waste issues will get worse in the future. Even in China, industrial waste was also the biggest worry for the future. Industrial waste issue with forestry declination and household waste issues in Japan, industrial waste issue with air pollution and water contamination issues in South Korea, and industrial waste issue with food safety and household waste issues in China, are the top three worries respectively.

\subsubsection{Environmental Sensitivity: From Past to the Present and to Future}

People's perception on environmental change, satisfaction with the environmental quality, and prediction for environment issues, were clarified above on three temporal scales. In this part, the MCA method is used to clarify the relationship among environmental sensitivity on these three temporal scales, as well as their relationship with surveyed countries or regions. Since the investigations of people's satisfactions and predictions are on a domestic level, the MCA analysis will only focus on people's evaluation on domestic environmental changes. The result of the analysis is shown in Figure 5.

According to the spatial position of variables in Figure 5, four groups can be generally distinguished. Environment was "improved", "satisfied” with the environment and environment will "improve dramatically" are located in the lower left quadrant, which represent the most positive evaluations of environmental change; Beijing and Hangzhou, together with "improved somewhat”, "satisfied somewhat”, environment will "improve” are located in upper left quadrant, which represent somewhat positive evaluations; "no change” in the past, 


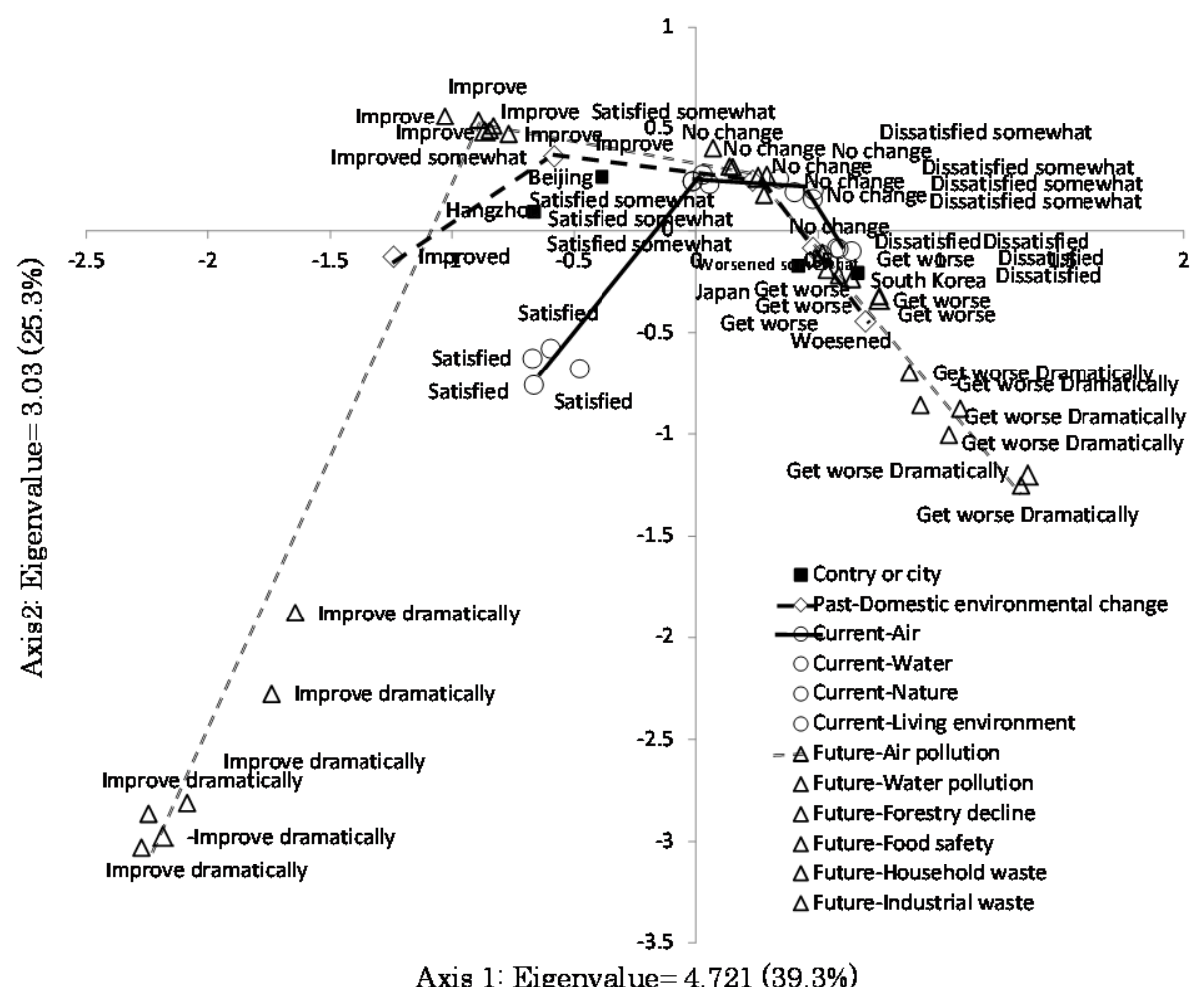

Axis 1: Eigenvalue $=4.721(39.3 \%)$

Figure 5. Environmental sensitivity on the temporal dimension.

"dissatisfied somewhat" and "no change" in the future are located in the upper right quadrant, which represent a medium evaluations; and Japan and South Korea together with "worsened" "dissatisfied somewhat" "get worse" and "get worse dramatically" are located in the lower right quadrant, which represent the most negative evaluations.

Based on the distribution of Figure 5, two tendencies are indicated: 1) people's environmental sensitivity on the temporal dimension has a consistency feature. People who believe the environmental quality improved in the past are inclined to be more satisfied with the present environment and also hold a positive prediction toward the future. Whereas people who believe the environmental quality got worse in the past are inclined to be dissatisfied with the present environment and also tend to believe that environmental issues will get even worse in the future. However, from the added line in Figure 5, we found that people's environmental perceptions of the past and environmental predictions of the future represent a higher consistency than with the satisfaction of the present. 2) The Japanese and South Koreans are pessimistic, while the Chinese are optimistic regarding changes to the environment. In Figure 5, Beijing and Hangzhou are close to the group of somewhat positive evaluations. Hangzhou is closest to the most positive evaluations distributed in the lower left quadrant. On the other side, Japan and South Korea are close to the group of somewhat negative evaluations. South Korea is closest to the most negative evaluations distributed in the lower right quadrant.

\subsection{The Relationship between Environmental Sensitivity and Demographic Factors}

In the previous sections, we analysed the spatial and temporal features of environmental sensitivity on a national level. In this section, we focus on the clarification of the relationship between environmental sensitivity and demographic factors within one country. We just focus on the relationship between environmental sensitivity to domestic environmental change on the past scale with demographic factors, and aim to clarify the attributive features of individuals who are more sensitive to environmental change of the past in the three countries.

In the analysis, age, education level and yearly household income are distributed into low, middle and high categories (more detail information see Table 4). And the evaluations on environmental change are also distributed into improved, no change and worsened categories. The relationship between environmental sensitivity 
Table 4. Classification standards and distributions in each category in surveyed regions.

\begin{tabular}{|c|c|c|c|c|c|}
\hline & & Japan (\%) & South Korea (\%) & Beijing (\%) & Hangzhou (\%) \\
\hline \multirow{2}{*}{ Gender } & Male & $49.4 \%$ & $48.7 \%$ & $50.9 \%$ & $51.0 \%$ \\
\hline & Female & $50.6 \%$ & $51.3 \%$ & $49.1 \%$ & $49.0 \%$ \\
\hline \multirow{3}{*}{ Low category } & Age & $20-34(14.8)$ & $19-34(27.7)$ & $18-34(40.1)$ & $18-34(33.1)$ \\
\hline & Education & $\begin{array}{l}\text { Elementary school/ } \\
\text { Junior high school (13.6) }\end{array}$ & $\begin{array}{l}\text { Elementary school/ } \\
\text { middle school (18.0) }\end{array}$ & $\begin{array}{l}\text { Less than one year/ } \\
\text { elementary school/ } \\
\text { middle school (33.6) }\end{array}$ & $\begin{array}{l}\text { Less than one year/ } \\
\text { elementary school/ } \\
\text { middle school (47.4) }\end{array}$ \\
\hline & Income & $\sim 4$ million yen (41.2) & 30 million won (24.5) & $\sim 40,000$ yuan $(42.7)$ & 40,000 yuan (27.9) \\
\hline \multirow{3}{*}{ Middle category } & Age & 35 - $54(24.4)$ & 35 - $54(35.4)$ & $35-54(29.8)$ & 35 - $54(32.4)$ \\
\hline & Education & High school (43.3) & High school (40.0) & High school (26.3) & High school (20.2) \\
\hline & Income & $\begin{array}{c}4 \text { - } 8 \text { million yen } \\
(38.2)\end{array}$ & $\begin{array}{c}30 \sim 90 \text { million won } \\
(26.2)\end{array}$ & $\begin{array}{c}40,000 \text { - 100,000 yuan } \\
\text { (34.7) }\end{array}$ & $\begin{array}{c}40,000-100,000 \text { yuan } \\
(44.6)\end{array}$ \\
\hline \multirow{3}{*}{ High category } & Age & Over 55 (60.9) & Over 55 (36.8) & Over 55 (30.1) & Over 55 (34.4) \\
\hline & Education & $\begin{array}{c}\text { Junior college/ } \\
\text { vocational school/ } \\
\text { university/graduate school } \\
\text { (43.2) }\end{array}$ & $\begin{array}{c}\text { Junior college/ } \\
\text { vocational school/ } \\
\text { college/graduate school } \\
\text { (42.0) }\end{array}$ & $\begin{array}{c}\text { Junior college/ } \\
\text { vocational school/ } \\
\text { college/graduate school } \\
\text { (40.1) }\end{array}$ & $\begin{array}{c}\text { Junior college/ } \\
\text { vocational school/ } \\
\text { college/graduate school } \\
\text { (32.4) }\end{array}$ \\
\hline & Income & 8 million yen $\sim(20.6)$ & 90 million won $\sim(49.3)$ & 100,000 yuan $\sim(22.6)$ & 100,000 yuan $\sim(27.6)$ \\
\hline
\end{tabular}

with demographic factors is analysed by the MCA method, and the results are shown in Figures 6(a)-(d).

In Figure 6(a), positive and medium evaluations are located in the upper right quadrant and the negative evaluation is located in the lower left quadrant. Individuals who are close to the negative evaluations are defined as more sensitive to environmental deterioration. For the four factors we selected, we found that females in Japan are more sensitive to the deterioration of the environment than males. Generally, in Japan, the order people are more sensitive to the deterioration of the environment. People with higher incomes and higher education are inclined to be more sensitive to the deterioration of environment.

In Figure 6(b), the positive and medium evaluations are located above Axis 1 and negative evaluation is located below Axis 1. From the location of variables in the figure, we found that in South Korea, middle-aged, middle-educated and middle-rich people are inclined to be more sensitive to the deterioration of the environment, while the younger and higher educated people are more inclined to give a positive evaluation of the changes in environmental quality. Similar to Japan, females are more sensitive to the deterioration of the environment.

In Figure 6(c), the positive evaluation is located on the left side of Axis 2, the medium evaluation located in the upper right quadrant, and the negative evaluation is located on in the lower right quadrant. From the location of variables in the figure, we found that education and income are generally positively related to sensitivity toward environmental deterioration. That is, with the increase of education and income, people are more sensitive to the deterioration of the environment. Age is generally negatively related to environmental sensitivity. Younger people are more sensitive to the deterioration of the environment. Differing from Japan and South Korea, males in Beijing are more inclined to be sensitive the deterioration of environment than females.

In Figure 6(d), the positive evaluation is located in the lower right quadrant, the medium evaluation is located in the lower left quadrant, and the negative evaluation is located in the upper left quadrant. The distribution of variables in Hangzhou is very similar to Beijing, in that education and income are positively related with environmental sensitivity and age is negatively related with environmental sensitivity. Younger, higher educated, and richer people are inclined to be more sensitive to the deterioration of environment. However, differing from Beijing, females in Hangzhou are more sensitive to the deterioration of the environment, which is consistent with the results from Japan and South Korea.

From the above results we clarified the features of individuals who are more sensitive to environmental 


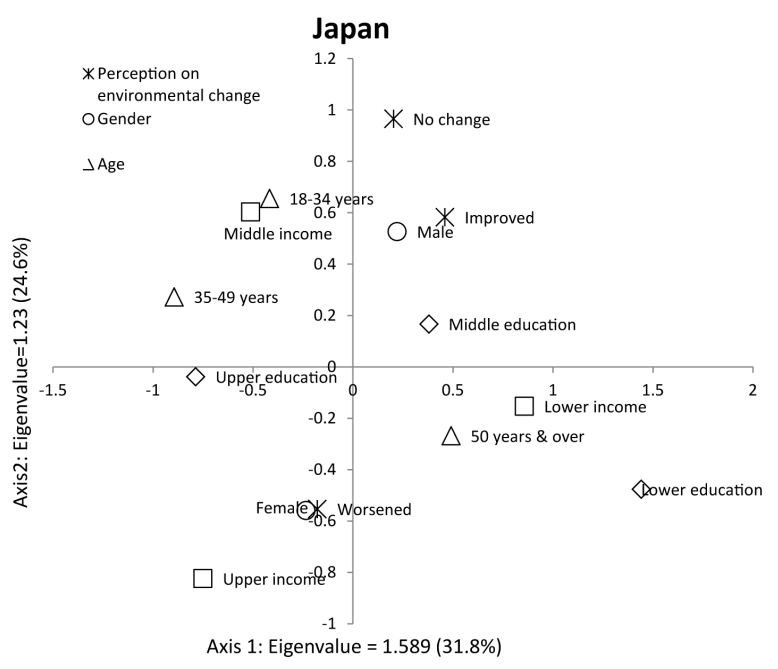

(a)

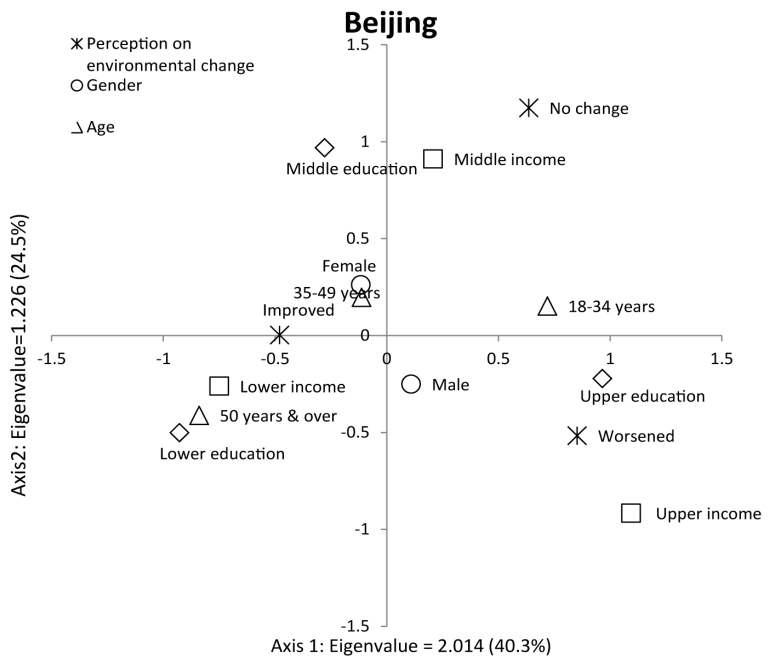

(c)

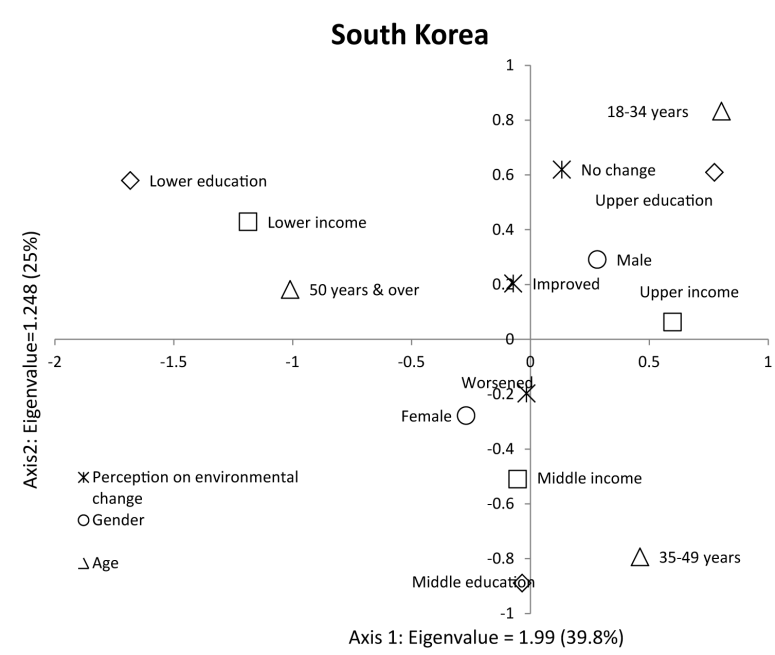

(b)

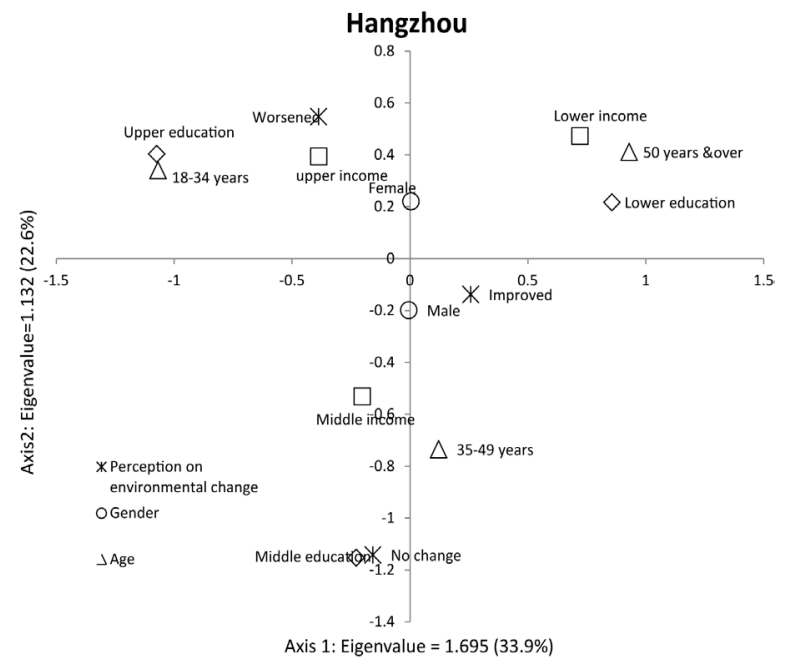

(d)

Figure 6. Relationship between demographic factors and environmental sensitivity in each country.

change in each region. In Japan, females, older people, and people with higher education and income are inclined to be more sensitive to the deterioration of environment. In South Korea, females and middle-aged, middle-educated and middle-rich people are inclined to be more sensitive to the deterioration of environment. In China, education and income are positively related to environmental sensitivity, while age is negatively related to environmental sensitivity. That is, richer, higher educated and younger people in China are more sensitive to the deterioration of environment. However, the influences of gender in the two cities of China are proved to be different. Differing from Japan, South Korea and Hangzhou, males in Beijing are more sensitive to the deterioration of environment than females.

\section{Discussion and Conclusion}

The purpose of this paper is to compare people's environmental sensitivity to environmental change in China, Japan and South Korea across spatial and temporal dimensions by analysing the data derived from a crossnational survey. Based on the 3907 cases collected from these three countries, the consistency of peoples' environmental consciousness on the temporal dimension and the gap on the spatial dimension were clarified.

The analysis on the temporal dimension of environmental sensitivity shows that people who give a positive/ negative evaluation toward the environmental change of the past, are also inclined to hold a positive/negative at- 
titude to the present and future. People's evaluation for the present is based on the experience of the past, and the prediction for the future is also derived from the feeling and experience of the past and present. The consistency feature of environmental sensitivity indicates that people's environmental consciousness has a stable nature.

The analysis on the spatial dimension of environmental sensitivity shows that people are more sensitive to the deterioration of the global environment rather the than domestic environment. One of the possible explanations for the gap between global and domestic environmental evaluations might be the influence of mass media. People's information regarding the global environment comes primarily from mass media which full of environmental challenges and irreversible influence. However, such challenges and influences are not tangible, immediate or visible in the course of day to day life. This might give people an illusion that environmental issues are not as much of an emergency as it described, or at least the environment around them is much better. This may become one of the reasons why the environment situation continues to worsen. To some extent, the more positive evaluations on domestic environment might also explain the high percentage of current satisfaction on the environmental quality, since they believe that they are enjoying a better environment than others.

From the responses of the three countries regarding environmental sensitivity, this research also indicates that the Japanese and South Koreans are pessimists and the Chinese are optimists when it comes to environmental quality and its change. Generally speaking, the Japanese hold a cautious evaluation of changes to environmental quality. More than half of the respondents in Japan believed that both the global and domestic environment worsened in the past several years, and only less than one fifth of the respondents believed that environmental issues will get better in the future. Comparatively, more than half of the Chinese respondents believed that both the global and domestic environment had improved in the past, and nearly half of the people believed the environmental issues will get better in the future. The Japanese pessimistic attitude reflects not only the evaluation to the environment, but other aspects as well. According to the data of the Japan Institute of Statistical Mathematics, in the past 20 years, the Japanese have held a more and more negative evaluation of Japanese society, their economy, their living standards, etc. This trend was described as the rapid spread of what might be called "the pessimistic view of society" (Japan Institute of Statistical Mathematics, 2011). However, in spite of the negative evaluations of the past and future, the Japanese hold high levels of satisfaction with the current environmental quality. However, also considered pessimist, South Korean's negative attitudes go through the whole temporal dimension.

Objective environment conditions may influence people's evaluations on the environment. In spite of the negative evaluation of the past and future, the Japanese show a very high level of satisfaction with the current environment. In Japan, the objective environmental quality of the present is much better and stable than that in China and South Korea, and hence the Japanese showed higher levels of satisfaction with the present environment. The stable environment may also explain why considerable proportion of the Japanese respondents chose "no change" regarding the future environment. The influence of objective environmental conditions can also be seen from the worries in China for the future. In Japan and South Korea, compared with other environmental issues, food safety is an issue that the least to be worried in the future. However, food safety is one of the top three worries in both cities of China. Such results may stem from the severe food safety reality in the present China.

About the relationship between demographic factors and environmental concern, it is a common place to conduct the research. However, it is still a place full of controversy, and needs to be further studied. The general conclusions are that the younger generation, women and those of a higher social class (indicated by higher education, income and occupation prestige) tend to be more concerned about environmental quality than others (Mohai \& Twight, 1987; Van Liere \& Dunlap, 1980; Dietz, Stern, Guagnano, 1998; etc.). However, the results show that under different social backgrounds, the influence of demographic factors is somewhat dissimilar. Females are supposed to be more sensitive to the deterioration of the environment. However, this was not verified in Beijing. The positive relationship between education and income with environment sensitivity is proved in China, while upper social class in Japan and middle social class in South Korea showed more environmental sensitivity. Older people in Japan, middle-aged people in South Korea and younger people in China showed more environmental sensitivity than others. Without further analysis we cannot provide more explanations for these differences. However, such differences are supposed to be particularly important. On the one side, these findings indicate that there are no universal conclusions about the influence of demographic factors that can be applicable to all cases, and on the other side, these findings may also reveal the influence of different social backgrounds to people's environmental consciousness. The results in this study provide a starting point, as well 
as beneficial reference for further and deeper analysis on these issues.

\section{Acknowledgements}

This research was partially supported by Grants-in-Aid for Scientific Research of Japan Society for the Promotion of Science, and the Grants-in-Aid for Academic Promotion, Graduate School of Culture and Information Science, Doshisha University.

\section{References}

Axelrod, L. (1994). Balancing Personal Needs with Environmental Preservation: Identifying the Values That Guide Decisions in Ecological Dilemmas. Journal of Social Issues, 50, 85-104. http://dx.doi.org/10.1111/j.1540-4560.1994.tb02421.X

Dietz, T., Stern, P. C., \& Guagnano, G. A. (1998). Social Structural and Social Psychological Bases of Environmental Concern. Environment and Behaviour, 30, 450-471. http://dx.doi.org/10.1177/001391659803000402

Disch, R. (1970). The Ecological Conscience: Values for Survival. Englewood Cliffs, NJ: Prentice-Hall.

Dunlap, R. E., \& Van Liere, K. D. (1978). The “New Environmental Paradigm”. The Journal of Environmental Education, 9 , 10-19. http://dx.doi.org/10.1080/00958964.1978.10801875

Inglehart, R. (1997). Modernization and Postmodernization: Cultural, Economic, and Political Change in 43 Societies (Vol. 19). Princeton, NJ: Princeton University Press.

Japan Institute of Statistical Mathematics (2011). The Surveys on the Japanese National Character. http://survey.ism.ac.jp/ks_e/index_e.html

Merchant, C. (1992). Radical Ecology: The Search for a Livable World. New York: Routledge, Chapman \& Hall.

Roth, C. E. (1968). On the Road to Conservation. Massachusetts Audubon, 38-41.

Roth, C. E. (1991). Towards Shaping Environmental Literacy for a Sustainable Future. Standardization News, 19 , 42-45.

Swan, J. A. (1971). Environmental Education: One Approach to Resolving the Environmental Crisis. Environment and Behavior, 3, 223-229. http://dx.doi.org/10.1177/001391657100300301

Mohai, P., \& Twight, B. (1987). Age and Environmentalism: An Elaboration of the Buttel Model Using National Survey Evidence. Social Science Quarterly, No. 68.

Stern, P. C. (1992). Psychological Dimensions of Global Environmental Change. Annual Review of Psychology, 43, $269-302$. http://dx.doi.org/10.1146/annurev.ps.43.020192.001413

Van Liere, K. D., \& Dunlap, R. E. (1980). The Social Bases of Environmental Concern: A Review of Hypotheses, Explanations and Empirical Evidence. Public Opinion Quarterly, 44, 181-197. http://dx.doi.org/10.1086/268583

Zheng, Y. (2010). Association Analysis on Pro-Environmental Behaviors and Environmental Consciousness in Main Cities of East Asia. Behaviormetrika, 37, 55-69. http://dx.doi.org/10.2333/bhmk.37.55

Zheng, Y. (2012). The East Asian Survey on People's Sense of Culture, Life and Environments (2010-2011). Doshisha Research Center for East Asian Studies. 